Research Article

\title{
Uncertainty-Based Trimmed Coefficient of Variation with Application
}

\author{
Ali Hussein Al-Marshadi, Muhammad Aslam ${ }^{D}$, and Alharbey Abdullah \\ Department of Statistics, Faculty of Science, King Abdulaziz University, Jeddah 21551, Saudi Arabia \\ Correspondence should be addressed to Muhammad Aslam; aslam_ravian@hotmail.com
}

Received 12 January 2021; Accepted 24 September 2021; Published 4 October 2021

Academic Editor: Parimala Mani

Copyright (C) 2021 Ali Hussein Al-Marshadi et al. This is an open access article distributed under the Creative Commons Attribution License, which permits unrestricted use, distribution, and reproduction in any medium, provided the original work is properly cited.

In this paper, the neutrosophic trimmed average, neutrosophic trimmed standard deviation, and neutrosophic trimmed coefficient of variation (NTCV) are introduced. The application of the proposed neutrosophic trimmed descriptive statistics is given with the help of measurement data. The comparisons of the proposed NTCV are compared with the existing neutrosophic coefficient of variation (NCV). From the comparisons, it is concluded that the proposed NTCV is more efficient than NCV in terms of consistency and measures of indeterminacy. Based on the study, it is recommended to apply the proposed NTCV in the industry when there is a need to make decisions on the basis of measurement data.

\section{Introduction}

The statistical methods and techniques are playing an important role in decision-making in all fields of social sciences, medical sciences, and industries. Among them, the average and coefficient of variation $(\mathrm{CV})$ have been widely used in decision-making in the presence of more than one characteristic. The average is used to select the variable of interest which is better on average, and CV is applied to check the consistency of that characteristic. For example, industrials are interested to make the decision about the product on the basis of measurements recorded by different operators. To make the decision, a more consistent operator is selected using the $\mathrm{CV}$. It is important to note that better on the average does not mean more consistent than the others. In other words, the CV tells about the variation in the data. Less the variation means better the data for the decision-making. A CV of less than $10 \%$ is considered very good and larger than $30 \%$ is not acceptable. The variation in the data can be reduced by omitting the outliers from the data. The outlier of the data can be removed from the data using the idea of the trimmed mean. In this method, a preselected percentage of the values are removed from the starting and ending of the ordered data. The use of the trimmed average is helpful to reduce the variation by removing extreme observations from the data. $\mathrm{Wu}$ and $\mathrm{Zuo}$ [1] proposed trimmed measures using the scale deviation method. Alkhazaleh and Razali [2] worked on estimation using the trimmed average. Yusof et al. [3] discussed various trimmed methods. Wang et al. [4] introduced the mean approach in medical science. Lugosi and Mendelson [5] introduced heavy-tailed distribution. More information about the application of trimmed measures can be seen in [6-10].

Uncertainty is defined as the lack of sureness about measurement, parameters, and observations. For example, measuring the water level, measuring rock joint roughness, and measuring the lifetime of a virus is done under uncertain environment. According to [11], "different sources of uncertainty may affect the quality of measurement results: environment, measurement setup, measuring instrument, appraiser, measuring object, measuring procedure, physical constants, the definition of the characteristic, software, and calculations." In case, when uncertainty is presented in the data, the fuzzy logic can be applied for the analysis of the data. The trimmed average under fuzzy logic can be applied to remove the extreme observations from the fuzzy data. The authors of [12-15] discussed the applications of trimmed average using fuzzy logic. More applications can be seen in $[16,17]$. 
Fuzzy logic is based on membership and nonmembership values. Neutrosophic logic is a general form of logic that deals with three measures, namely, the measure of truth (membership), the measure of falsehood (nonmembership), and the measure of indeterminacy. The fuzzy logic is a special case of neutrosophic logic, see [18]. The information about the measure of indeterminacy can be obtained from the neutrosophic logic. The neutrosophic logic has been applied in a variety of fields, see [19-21]. Using the idea of neutrosophic logic, neutrosophic statistics which is the extension of classical statistics was introduced by [22]. The methods to analyze the neutrosophic data were discussed in $[23,24]$. Aslam [25] introduced the neutrosophic coefficient of variation. Aslam and Bantan [26] introduced a measurement system using neutrosophic statistics. More information on dealing neutrosophic numbers can be seen in [27-29].

As mentioned earlier, the trimmed average is a useful technique to reduce the variation by removing the extreme observations from the data. In this method, a small percentage of values are removed to minimize the variation in the data. The trimmed average helps to remove the outliers from the data before calculating the traditional average. The coefficient of variation under neutrosophic statistics is known as the neutrosophic coefficient of variation (NCV) and the coefficient of variation using the trimmed average under neutrosophic statistics is known as the neutrosophic trimmed standard deviation (NTSD). The coefficient of variation using trimmed average under classical is called the trimmed coefficient of variation. Aslam [25] introduced NCV. By exploring the literature and best of our knowledge, there is no work on neutrosophic trimmed average, neutrosophic trimmed standard deviation (NTSD), and NTCV. In this paper, the introduction of average, standard deviation, and coefficient of variation using the neutrosophic statistics will be given. In addition, we will give the application of the proposed NTCV using the measurement data from the industry. It is expected that the proposed NTCV will be helpful to increase the consistency as compared to NCV. Furthermore, the proposed NTCV will be helpful to minimize the measure of indeterminacy.

\section{Methodology}

Let $I_{N} \epsilon\left[I_{L}, I_{U}\right]$ be an indeterminacy interval associated with neutrosophic random number $X_{N i}=X_{L i}+X_{U i} I_{N}\left(i=1,2,3, \ldots, n_{N}\right) \quad$ of size $n_{N} \epsilon\left[n_{L}, n_{U}\right]$, where $X_{L}, n_{L}$ and $X_{U}, n_{U}$ are the lower and upper values, respectively. The basic operations such as multiplication, division, and inverse of these neutrosophic numbers can be seen in $[23,24]$. Suppose a data analyst has a neutrosophic sample $n_{N} \epsilon\left[n_{L}, n_{U}\right]$ and he is interested to find $\alpha \%$ neutrosophic trimmed average (NTA). Suppose that $X_{L}$ and $X_{U}$ denote the lower and upper values of an indeterminate interval of measurement parts. The trimmed observation is denoted by $k_{N}=n_{N} \alpha$, where $\alpha$ is the percentage of values trimmed from the data. Suppose that $R_{N}=n_{N}-$ $n_{N} \alpha$ shows the difference between the total observation and trimmed observations. The following process can be adopted to calculate $\alpha \%$ neutrosophic trimmed average.

Step 1: arrange $X_{L}$ and $X_{U}$ observations in the ascending order

Step 2: trim $k_{N} \epsilon\left[k_{L}, k_{U}\right]$ observations at both ends of arranged data, where $k_{N}=n_{N} \alpha$

Step 3: compute NTA of remaining observations, $R_{N}=n_{N}-n_{N} \alpha, R_{N} \epsilon\left[R_{L}, R_{U}\right]$

The neutrosophic trimmed average, say $\bar{T}_{L}$ of values $X_{L}$, is calculated as

$$
\bar{T}_{L}=\frac{1}{R_{L}} \sum_{i=k_{L}+1}^{n_{L}-k_{L}} X_{L i}
$$

where index of summation runs from the lower value of $k_{N}$ to the lower value of $R_{N}$.

The neutrosophic trimmed average, say $\bar{T}_{U}$ of values $X_{U}$, is calculated as

$$
\bar{T}_{U}=\frac{1}{R_{U}} \sum_{i=k_{U}+1}^{n_{U}-k_{U}} X_{U i}
$$

where index of summation runs from the upper value of $k_{N}$ to the upper value of $R_{N}$.

The neutrosophic trimmed average, say $\bar{X}_{N i}$, using equations (1) and (2), is calculated by

$$
\bar{X}_{N i}=\frac{1}{R_{L}} \sum_{i=k_{L}+1}^{n_{L}-k_{L}} X_{L i}+\frac{1}{R_{U}} \sum_{i=k_{U}+1}^{n_{U}-k_{U}} X_{U i} I_{\bar{X} N} ; I_{\bar{X} N} \epsilon\left[I_{\bar{X} L}, I_{\bar{X} U}\right] .
$$

The neutrosophic trimmed sum of the square of observations from $\bar{X}_{N i}$ is calculated by

$$
\sum_{i=k_{U}+1}^{n_{U}-k_{U}}\left(X_{N i}-\bar{X}_{N i}\right)^{2}=\sum_{i=k_{U}+1}^{n_{U}-k_{U}}\left[\begin{array}{r}
\min \left(\begin{array}{c}
\left(X_{L i}+X_{U i} I_{L}\right)\left(\bar{X}_{L}+\bar{X}_{U} I_{L}\right),\left(X_{L i}+X_{U i} I_{L}\right)\left(\bar{X}_{L}+\bar{X}_{U} I_{U}\right), \\
\left(X_{L i}+X_{U i} I_{U}\right)\left(\bar{X}_{L}+\bar{X}_{U} I_{L}\right),\left(X_{L i}+X_{U i} I_{U}\right)\left(\bar{X}_{L}+\bar{X}_{U} I_{U}\right)
\end{array}\right) \\
\max \left(\begin{array}{c}
\left(X_{L i}+X_{U i} I_{L}\right)\left(\bar{X}_{L}+\bar{X}_{U} I_{L}\right),\left(X_{L i}+X_{U i} I_{L}\right)\left(\bar{X}_{L}+\bar{X}_{U} I_{U}\right), \\
\left(X_{L i}+X_{U i} I_{U}\right)\left(\bar{X}_{L}+\bar{X}_{U} I_{L}\right),\left(X_{L i}+X_{U i} I_{U}\right)\left(\bar{X}_{L}+\bar{X}_{U} I_{U}\right)
\end{array}\right)
\end{array}\right], I_{N} \epsilon\left[I_{L}, I_{U}\right],
$$


where $\bar{X}_{L}=1 / n_{L} \sum_{i=1}^{n_{L}} X_{L i}$ and $\bar{X}_{U}=1 / n_{U} \sum_{i=1}^{n_{U}} X_{U i}$

The neutrosophic trimmed standard deviation (NTSD), say $s_{N T}$, is given by

$$
s_{N T}=\sqrt{\frac{1}{R_{N}} \sum_{i=k_{N}+1}^{n_{N}-k_{N}}\left(X_{N i}-\bar{X}_{N i}\right)^{2} .}
$$

The neutrosophic trimmed coefficient of variation (NTCV) tells about the consistency and is computed by

$$
C V_{N T}=\frac{s_{N T}}{\bar{X}_{N i}} \times 100
$$

\section{Application Using Measurement Data}

Now, we present the case study from the automotive industry in Kachiran Company in Asia, see [30], for more details. The company is a manufacturing housing clutch used as automobile parts. To make a better decision about the performance of these parts, the company needs the measurements of these parts. The decision about the performance depends on the consistency of the operators. The operators working in the company have the instruction to measure the length of the parts. The measurements of these parts cannot be recorded completely; therefore, the measurement observations are neutrosophic. The measurements in $\mathrm{mm}$ by three operators are shown in Table 1 .

From Table 1, it can be seen that the use of classical statistics may mislead the managers in decision-making. Therefore, the consistency of the operators in measuring will be discussed with the help of the proposed methods. Let $\alpha=$ $1 \%$ and $n_{N} \in[10,10]$. The application of the proposed method to find NTCV for operator 1 is stated as follows (Tables 2 and $3)$.

Step 1: arrange $X_{L}$ and $X_{U}$ observations of operator 1 in the ascending order as shown in Table 2.

Step 2: trim $k_{N}=1$ observations at both ends of arranged data, where $k_{N}=10 \times 0.1$. The remaining data are given in Table 3.

Step 3: compute NTA of remaining observations, $R_{N}=10-2=8$.

The neutrosophic trimmed average of values $X_{L}$ is calculated as

$$
\bar{T}_{L}=\frac{1}{8} \sum_{i=2}^{8} X_{L i}=62.12 .
$$

The neutrosophic trimmed average of values $X_{U}$ is calculated as

$$
\bar{T}_{U}=\frac{1}{8} \sum_{i=2}^{8} X_{U i}=62.24 .
$$

The neutrosophic trimmed average is defined by

$$
\bar{X}_{N i}=62.12+62.24 I_{N} ; I_{N} \epsilon[0,0.0019] .
$$

The neutrosophic trimmed sum of the square is calculated by

$$
\sum_{i=2}^{8}\left(X_{N i}-\bar{X}_{N i}\right)^{2}=[0.0264,0.1059] .
$$

The neutrosophic trimmed standard deviation (NTSD), say $s_{N T}$, is given by

$$
s_{N T}=\sqrt{\frac{1}{8} \sum_{i=2}^{8}\left(X_{N i}-\bar{X}_{N i}\right)^{2}}=[0.0575,0.1150] .
$$

The neutrosophic trimmed coefficient of variation (NTCV) tells about the consistency and computed by

$$
C V_{N T}=\frac{[0.0575,0.1150]}{[62.12,62.24]} \times 100=[0.0924,0.1851] \text {. }
$$

The values of NTCV for other operators can be calculated in the same way as for operator 1 . The neutrosophic descriptive statistics for three operators are shown in Table 4 . From the first column of Table 4, it can be seen that, on average in measurement, operator 2 is better than other operators. We also note that the indeterminacy interval of operator 3 is smaller than other operators. Therefore, operator 3 is more consistent in measuring the length of housing clutch parts. Based on this study, it is concluded that the management can make the decision about the product on the basis of measurement recorded by operator 3 .

\section{Comparative Study}

Aslam [25] introduced the neutrosophic coefficient of variation $(\mathrm{NCV})$ under the neutrosophic statistics. In this section, we will discuss the advantages of the proposed NTCV with NCV. Note here that the proposed NTCV reduces to the existing NCV when no observation is trimmed from the data $(\alpha=0 \%)$. To show the efficiency of the proposed NTCV over NCV, we will consider the same descriptive neutrosophic statistics of measurement data are presented in the last section. The NCV and NTCV for three operators are shown in Table 5. From column four of Table 5, it can be noted that the values of NTCV from the proposed method are smaller than the existing NCV which indicates that the proposed NTCV is more consistent in measurement as compared to NCV. For example, for the measurement data given by operator 3 , the indeterminate interval is from 0.0826 to 0.1695 . On the contrary, this interval from the existing NCV is from 0.1029 to 0.2045 . From Table 5, it can also be noted that the use of the proposed method increases the efficiency of the values of the coefficient of variation. From this study, it is concluded that the proposed NTCV is smaller than the existing NCV. We conclude that the proposed method is helpful to increase the consistency of measurement. The neutrosophic forms of NCV and NTCV along with the measures of indeterminacy are placed in Table 6. The first values in neutrosophic form denote the determined values under classical statistics and the second 
TABLe 1: The real example data.

\begin{tabular}{lccc}
\hline Part no. & \multicolumn{3}{c}{ Operators } \\
\hline 1 & 1 & 2 & 3 \\
2 & {$[62.14,62.26]$} & {$[62.09,62.21]$} & {$[62.09,62.21]$} \\
3 & {$[62.13,62.25]$} & {$[62.13,62.25]$} & {$[62.13,62.25]$} \\
4 & {$[62.05,62.17]$} & {$[62.05,62.17]$} & {$[62.04,62.16]$} \\
5 & {$[62.11,62.23]$} & {$[62.11,62.23]$} & {$[62.11,62.23]$} \\
6 & {$[62.19,62.31]$} & {$[62.06,62.18]$} & {$[62.19,62.31]$} \\
7 & {$[62.06,62.18]$} & {$[62.08,62.20]$} & {$[62.06,62.18]$} \\
8 & {$[62.07,62.19]$} & {$[62.14,62.26]$} & {$[62.07,62.19]$} \\
9 & {$[62.14,62.26]$} & {$[62.24,62.36]$} & {$[62.14,62.26]$} \\
10 & {$[62.24,62.36]$} & {$[62.22,62.34]$} & {$[62.23,62.35]$} \\
\hline
\end{tabular}

TABLE 2: Observations of operator 1.

\begin{tabular}{lllllllllll}
\hline$X_{L}$ & 62.05 & 62.06 & 62.07 & 62.11 & 62.13 & 62.14 & 62.14 & 62.19 & 62.22 & 62.24 \\
\hline$X_{U}$ & 62.17 & 62.18 & 62.19 & 62.23 & 62.25 & 62.26 & 62.26 & 62.31 & 62.34 & 62.36 \\
\hline
\end{tabular}

TABle 3: Trimmed observations of operator 1.

\begin{tabular}{lllllllll}
\hline$X_{L}$ & 62.06 & 62.07 & 62.11 & 62.13 & 62.14 & 62.14 & 62.19 & 62.22 \\
\hline$X_{U}$ & 62.18 & 62.19 & 62.23 & 62.25 & 62.26 & 62.26 & 62.31 & 62.34 \\
\hline
\end{tabular}

TABle 4: Neutrosophic descriptive statistics.

\begin{tabular}{lcccc}
\hline Operators & $\bar{X}_{N i}$ & $s_{N T}$ & $C V_{N T}$ & Range \\
\hline 1 & {$[62.12,62.24]$} & {$[0.0575,0.1150]$} & {$[0.0924,0.1851]$} & 0.0927 \\
2 & {$[62.12,62.26]$} & {$[0.0514,0.1078]$} & {$[0.0826,0.1731]$} & 0.0905 \\
3 & {$[62.12,62.24]$} & {$[0.0526,0.1053]$} & {$[0.0845,0.1695]$} & 0.085 \\
\hline
\end{tabular}

TABLE 5: The comparison in NCV and NTCV.

\begin{tabular}{|c|c|c|c|c|}
\hline Operators & $\mathrm{NCV}$ & Status & NTCV & Status \\
\hline 1 & {$[0.1003,0.2011]$} & Good & {$[0.0924,0.1851]$} & Very good \\
\hline 2 & {$[0.1013,0.5628]$} & Not acceptable & {$[0.0826,0.1731]$} & Very good \\
\hline 3 & {$[0.1020,0.2045]$} & Good & {$[0.0845,0.1695]$} & Very good \\
\hline
\end{tabular}

TABLE 6: The comparison in NCV and NTCV.

\begin{tabular}{lcc}
\hline Operators & Neutrosophic form of NCV & Neutrosophic form of NTCV \\
\hline 1 & $0.1003+0.2011 I_{N}, I_{N} \epsilon[0,0.5012]$ & $0.0924+0.1851 I_{N}, I_{N} \epsilon[0,0.5]$ \\
2 & $0.1013+0.5628 I_{N}, I_{N} \epsilon[0,0.82]$ & $0.0826+0.1731 I_{N}, I_{N} \epsilon[0,0.5228]$ \\
3 & $0.1020+0.2045 I_{N}, I_{N} \epsilon[0,0.5012]$ & $0.0845+0.1695 I_{N}, I_{N} \epsilon[0,0.5]$ \\
\hline
\end{tabular}

part is indeterminate parts. For example, in neutrosophic form $0.0826+0.1731 I_{N}, I_{N} \epsilon[0,0.5228]$, the value 0.0826 presents the value of the coefficient of variation $(\mathrm{CV})$ for classical statistics. The value $0.1731 I_{N}, I_{N} \epsilon[0,0.5228]$, is the indeterminate part with the measure of indeterminacy $(0.1731-0.0826) / 0.1731=0.5228$. We note that the measure of indeterminacy from the existing method is given by [25] is 0.82 . From this study, it is concluded that the proposed method is helpful to minimize the measure of indeterminacy. We also compared the results of the proposed study with interval statistics. The interval statistics used intervals in order to capture the data inside the intervals. Therefore, the interval statistics tells the values of NTCV from 0.0826 to 0.173 without giving any information about the measure of indeterminacy. Therefore, it is concluded that the proposed NTCV is more efficient in measure of indeterminacy than the existing CV proposed by [25] and interval statistics. 


\section{Concluding Remarks}

In this paper, the neutrosophic trimmed average, neutrosophic trimmed standard deviation, and neutrosophic trimmed coefficient of variation (NTCV) were introduced. The application of the proposed neutrosophic trimmed descriptive statistics was given with the help of measurement data. The comparisons of the proposed NTCV are compared with the existing neutrosophic coefficient of variation (NCV). From this study, it is can be seen that the proposed NTCV is more efficient than NCV in terms of measures of indeterminacy. In addition, it can be seen that the proposed NTCV reduces the variation in the measurement data. The proposed NTCV can be applied for the decision-making in the industry when the data are obtained from the measurement having the neutrosophy. The other trimmed statistical methods under neutrosophic statistics can be considered as future research.

\section{Data Availability}

The data used to support the findings of the study are given within the article.

\section{Conflicts of Interest}

The authors declare that they have no conflicts of interest.

\section{Acknowledgments}

This work was funded by the Deanship of Scientific Research (DSR), King Abdulaziz University, Jeddah, under Grant no. D-104-130-1441. The author, therefore, gratefully acknowledge the DSR technical and financial support.

\section{References}

[1] M. Wu and Y. Zuo, "Trimmed and Winsorized means based on a scaled deviation," Journal of Statistical Planning and Inference, vol. 139, no. 2, pp. 350-365, 2009.

[2] A. Alkhazaleh and A. Razali, "New technique to estimate the asymmetric trimming mean," Journal of Probability and Statistics, vol. 2010, Article ID 739154, 2010.

[3] Z. M. Yusof, S. Abdullah, S. S. S. Yahaya, and A. R. Othman, "Testing the equality of central tendency measures using various trimming strategies," African Journal of Mathematics and Computer Science Research, vol. 4, pp. 32-38, 2011.

[4] M.-D. Wang, J. Liu, G. Molenberghs, and C. Mallinckrodt, "An evaluation of the trimmed mean approach in clinical trials with dropout," Pharmaceutical Statistics, vol. 17, no. 3, pp. 278-289, 2018.

[5] G. Lugosi and S. Mendelson, "Mean estimation and regression under heavy-tailed distributions: a survey," Foundations of Computational Mathematics, vol. 19, no. 5, pp. 1145-1190, 2019.

[6] R. Oten and R. J. P. De Figueiredo, "Adaptive alpha-trimmed mean filters under deviations from assumed noise model," IEEE Transactions on Image Processing, vol. 13, no. 5, pp. 627-639, 2004.

[7] A. Brik, M. Goddi, J. Dhahri, and N. B. Fredj, "Assessing process capability index using sampling plan in the presence of measurement system errors," The International Journal of
Advanced Manufacturing Technology, vol. 102, no. 9-12, pp. 3301-3313, 2019.

[8] Z. Zhao, D. Ding, Y. Fu, J. Xu, and J. Han, “A hybrid approach for measurement thickness of complex structural parts using ultrasonic inspection and on-machine probing," The International Journal of Advanced Manufacturing Technology, vol. 103, no. 9-12, pp. 4777-4785, 2019.

[9] A. García-Pérez, "An approximation to the small sample distribution of the trimmed mean for Gaussian mixture models," in Strengthening Links Between Data Analysis and Soft Computing, pp. 115-122, Springer, Berlin, Germany, 2015.

[10] H. Liu, R. Yang, P. Wang, J. Chen, H. Xiang, and G. Chen, "Measurement point selection and compensation of geometric error of NC machine tools," The International Journal of Advanced Manufacturing Technology, vol. 108, no. 11-12, pp. 3537-3546, 2020.

[11] S. F. Beckert and W. S. Paim, "Critical analysis of the acceptance criteria used in measurement systems evaluation," International Journal of Metrology and Quality Engineering, vol. 8, p. 23, 2017.

[12] A. Hussain, Q. Javaid, and M. Siddique, "Impulse noise removal using fuzzy logic and alpha-trimmed mean," in Proceedings of the 2011 IEEE International Conference on Signal and Image Processing Applications (ICSIPA), pp. 216-220, Kuala Lumpur, Malaysia, November 2011.

[13] T. Veerakumar, S. Esakkirajan, and I. Vennila, "Combined fuzzy logic and unsymmetric trimmed median filter approach for the removal of high density impulse noise," WSEAS Transactions on Signal Processing, vol. 8, pp. 32-42, 2012.

[14] S. Vijaya Kumar and C. Nagaraju, "A fast adaptive fuzzy unsymmetric trimmed mean filter for removal of impulse noise from digital images," in Proceedings of the International Conference on Computing and Communication Systems, pp. 137-146, Shillong, India, April 2018.

[15] V. V. Vela-Rincón, D. Mújica-Vargas, M. Mejía Lavalle, and A. Magadán Salazar, "Spatial \$\$ $\$$ alpha \$ \$-trimmed fuzzy C-means algorithm to image segmentation," in Proceedings of the Mexican Conference on Pattern Recognition, pp. 118-128, Morelia, Mexico, June 2020.

[16] M. Asplund and J. Lin, "Evaluating the measurement capability of a wheel profile measurement system by using GR\&R," Measurement, vol. 92, pp. 19-27, 2016.

[17] K. Mittal, P. C. Tewari, and D. Khanduja, "On the fuzzy evaluation of measurement system analysis in a manufacturing and process industry environment: a comparative study," Management Science Letters, vol. 8, pp. 201216, 2018.

[18] F. Smarandache, Neutrosophy. Neutrosophic Probability, Set, and Logic, ProQuest Information \& Learning, vol. 105, pp. 118-123, American Research Press, Ann Arbor, MI, USA, 1998.

[19] R. Alhabib and A. Salama, "Using moving averages to pave the neutrosophic time series," International Journal of $\mathrm{Neu}$ trosophic Science, vol. 3, pp. 14-20, 2020.

[20] S. K. Das and S. Edalatpanah, "A new ranking function of triangular neutrosophic number and its application in integer programming," International Journal of Neutrosophic Science, vol. $4,2020$.

[21] P. Schweizer, "Uncertainty: two probabilities for the three states of neutrosophy," International Journal of Neutrosophic Science, vol. 2, pp. 18-26, 2020. 
[22] F. Smarandache, "Introduction to neutrosophic statistics: infinite study," 2014, https://arxiv.org/ftp/arxiv/papers/1406/ 1406.2000.

[23] J. Chen, J. Ye, and S. Du, "Scale effect and anisotropy analyzed for neutrosophic numbers of rock joint roughness coefficient based on neutrosophic statistics," Symmetry, vol. 9, no. 10, p. 208, 2017.

[24] J. Chen, J. Ye, S. Du, and R. Yong, "Expressions of rock joint roughness coefficient using neutrosophic interval statistical numbers," Symmetry, vol. 9, no. 7, p. 123, 2017.

[25] M. Aslam, "A new method to analyze rock joint roughness coefficient based on neutrosophic statistics," Measurement, vol. 146, pp. 65-71, 2019.

[26] M. Aslam and R. A. Bantan, "A study on measurement system analysis in the presence of indeterminacy," Measurement, vol. 166, Article ID 108201, 2020.

[27] A. Kumar, B. Sah, A. R. Singh et al., "A review of multi criteria decision making (MCDM) towards sustainable renewable energy development," Renewable and Sustainable Energy Reviews, vol. 69, pp. 596-609, 2017.

[28] A. Hussain and H.-M. Kim, "Goal-programming-based multi-objective optimization in off-grid microgrids," Sustainability, vol. 12, no. 19, p. 8119, 2020.

[29] T. Yang, Y. Kuo, D. Parker, and K. H. Chen, "A multiple attribute group decision making approach for solving problems with the assessment of preference relations," Mathematical Problems in Engineering, vol. 2015, Article ID 849897, 2015.

[30] A. Kazemi and V. Hajipour, "A fuzzy approach to increase accuracy and precision in measurement system analysis," Economic Computation \& Economic Cybernetics Studies \& Research, vol. 47, pp. 171-187, 2013. 